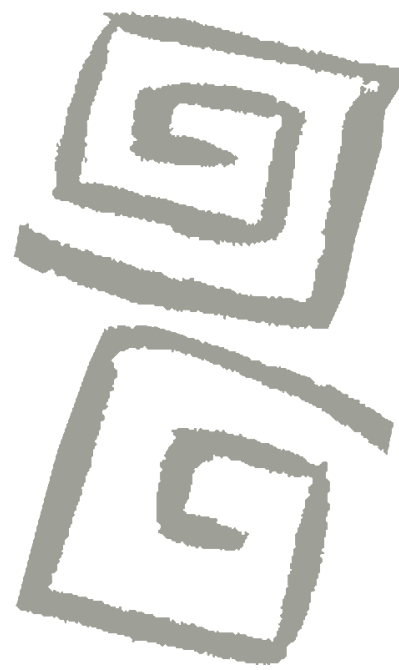

\title{
Prevalencia y evolución del uso de casco en ocupantes de motocicletas en una ciudad argentina (Mar del Plata, 2006-2014)
}

\author{
Prevalence and evolution of helmet use in \\ motorcycle riders in an Argentine city (Mar del \\ Plata, 2006-2014)
}

Jeremías David Tosi', Ruben Daniel Ledesma², Fernando Martín Poó ${ }^{3}$, Silvana Andrea Montes ${ }^{4}$, Soledad Susana López ${ }^{5}$

'Licenciado en Psicología. Becario Doctoral, Consejo Nacional de Investigaciones Científicas y Técnicas (CONICET), Universidad Nacional de Mar del Plata, Argentina.

jeremiastosi@gmail.com

${ }^{2}$ Licenciado en Psicología, Doctor en Psicología. Investigador Categoría Independiente, Consejo Nacional de Investigaciones Científicas y Técnicas (CONICET). Profesor Titular Interino, Universidad Nacional de Mar del Plata, Argentina. rdledesma@conicet.gov.ar

${ }^{3}$ Licenciado en Psicología, Doctor en Psicología. Investigador Asistente, Consejo Nacional de Investigaciones Científicas y Técnicas (CONICET). Ayudante de Trabajos Prácticos, Universidad Nacional de Mar del Plata, Argentina.

poo.fernando@gmail.com

${ }^{4}$ Licenciada en Psicología, Doctora en Psicología. Becaria posdoctoral, Consejo Nacional de Investigaciones Científicas y Técnicas (CONICET).

Ayudante de Trabajos Prácticos, Universidad Nacional de Mar del Plata, Argentina. silvanamontes3@gmail.com

${ }^{5}$ Licenciada en Psicología. Becaria Doctoral, Consejo Nacional de Investigacione Científicas y Técnicas (CONICET). Ayudante de Trabajos Prácticos, Universidad Nacional de Mar del Plata, Argentina.

soledadslopez@live.com.ar
RESUMEN Los siniestros viales que involucran a motociclistas conforman un problema creciente en los países de ingresos bajos y medios. La medida de protección por excelencia en estos usuarios viales es el casco. Sin embargo, una proporción considerable de motociclistas no lo usa. El objetivo de este trabajo es informar la evolución del uso de casco durante el periodo 2006-2014 en una ciudad de Argentina, y conocer los factores asociados durante el año 2014. La muestra incluye más de 6.900 observaciones de motociclistas, registradas en los años $2006(n=962), 2008(n=977), 2012(n=2.542)$ y $2014(n=2.466)$. Los datos indican un aumento progresivo del uso de casco a través del tiempo, aunque se sostienen las diferencias por género y función. Los factores asociados al uso de casco en conductores durante el año 2014 fueron el uso en pasajeros, el tipo de moto, la presencia de patente y el género. Aunque los resultados son positivos, conviene alertar sobre las consecuencias negativas del aumento del parque de motos.

PALABRAS CLAVES Accidentes de Tránsito; Motocicletas; Prevención de Accidentes; Argentina.

\begin{abstract}
Traffic collisions involving motorcyclists are a growing problem in low and middle income countries. Helmet use is the foremost protective measure for this group of road users, however many riders do not wear them. The objective of the present study is to report the changes in helmet use during the period 2006-2014 in an Argentine city and discover associated factors for the year 2014. The sample includes more than 6,900 observations of motorcyclists carried out during the years $2006(n=962), 2008(n=977)$, $2012(n=2,542)$, and $2014(n=2,466)$. The data indicates a progressive increase in helmet use over time, but differences due to gender and type of rider remain. Factors associated to helmet use in motorcycle drivers during 2014 were: passenger helmet use, motorcycle type, license plate use and gender. Although the results are positive, it is necessary to be attentive to the negative consequences of the growing fleet of motorcycles.
\end{abstract}

KEY WORDS Accidents, Traffic; Motorcycles; Accident Prevention; Argentina. 


\section{INTRODUCCIÓN}

Según la Organización Mundial de la Salud (OMS) las lesiones no intencionales provocadas por el tránsito constituyen un problema de salud pública que afecta a la población mundial, aunque su prevalencia varía en función del país y el tipo de usuario vial ${ }^{(1)}$. En su Informe sobre la situación mundial de la seguridad vial, la OMS estima que más del $80 \%$ de las muertes causadas por el tránsito ocurren en los países de ingresos bajos y medios. Entre los usuarios viales más afectados, actualmente es posible advertir el aumento progresivo de siniestros viales que involucran a motociclistas ${ }^{(1)}$. Un factor que incide en esta situación corresponde al incremento evidente del parque de $\operatorname{motos}^{(1),(2)}$.

Argentina no es ajena a esta situación. La moto se ha convertido en el segundo modo de transporte urbano, después del automóvil. El uso de moto ha crecido un $72,6 \%$ entre 2008 y $2011^{(2)}$. Según la Agencia Nacional de Seguridad Vial, en ese mismo periodo, la participación de motociclistas en colisiones de tránsito aumentó un $54 \%$. Un dato relevante es que, entre los motociclistas fallecidos por lesiones de tránsito, solo un $25 \%$ usaba casco en el momento de la colisión ${ }^{(2)}$.

Un aspecto que incrementa la mortalidad de los siniestros viales en motociclistas es que las lesiones más comunes se producen en la cabeza ${ }^{(3),(4)}$. Para este grupo, este tipo de lesiones representa la principal causa de muerte ${ }^{(5)}$. Algunos estudios estiman que aproximadamente el $80 \%$ de los motociclistas fallecidos después de una colisión de tránsito sufrieron previamente lesiones en la cabeza ${ }^{(6)}$. Por su parte, en aquellos motociclistas que sobrevivieron a ese tipo de lesiones, se destacan daños faciales que conllevan perjuicios secundarios (por ejemplo, problemas emocionales debido a cuestiones estéticas o mayores posibilidades de padecer trastorno por estrés postraumático ${ }^{(7)}$.

Por estas razones, el uso de casco es la medida de autoprotección por excelencia para los motociclistas ${ }^{(8)}$. Los estudios muestran que cuando este dispositivo de seguridad es utilizado correctamente reduce el riesgo de lesiones graves en más de un $70 \%$ y el riesgo de muerte en un $40 \%{ }^{(1)}$. Algunas investigaciones indican que el uso de casco también se asocia con una disminución en el tiempo de hospitalización y en los costos económicos en salud ${ }^{(9)}$. Por su parte, la falta de uso de casco se asocia a una mayor frecuencia y severidad de lesiones, posibles secuelas, hospitalizaciones más prolongadas, incremento de la mortalidad y costos hospitalarios significativamente más altos ${ }^{(10)}$. No obstante, el uso de casco sigue siendo irregular en muchos países, incluyendo Argentina ${ }^{(6),(11)}$.

Las bajas tasas de utilización del casco han Ilevado a estudiar posibles factores personales y contextuales asociados al comportamiento de uso. En cuanto a los factores personales, se han estudiado tanto aspectos sociodemográficos como psicológicos. Por ejemplo, investigaciones previas muestran un menor uso entre los conductores jóvenes, de sexo masculino, que conducen sin licencia o en vehículos sin patente y que poseen experiencia previa de siniestros ${ }^{(6),(12),(13),(14)}$. Por otro lado, las actitudes y creencias sobre el uso de casco también cumplen un rol importante ${ }^{(12)}$. Las creencias sobre la utilidad del uso de casco como medida para prevenir lesiones son facilitadoras del uso, mientras que la percepción de incomodidad resulta una barrera importante para su utilización ${ }^{(15),(16)}$. En un trabajo previo ${ }^{(17)}$, hemos mostrado que las actitudes implícitas y explícitas negativas hacia este dispositivo se asocian a un menor uso. En particular, el componente "emocional" de las actitudes (percepción de incomodidad, displacer en el uso, etc.) cumple un papel muy importante en la predicción de la conducta de uso, siendo más relevante que el componente cognitivo-racional (valoración de la utilidad del casco como medida de seguridad).

Las variables familiares y la influencia de pares son otros aspectos que se han considerado en la bibliografía ${ }^{(6),(18),(19)}$. La influencia de pares y las normas subjetivas compartidas pueden incidir sobre el uso, tanto de manera positiva como de manera negativa. Por ejemplo, cuando la cultura familiar o del grupo de pares le otorga un rol 
importante a la seguridad, los motociclistas se ven más inclinados a usar el $\operatorname{casco}^{(6)}$. $\mathrm{Al}$ contrario, cuando los jóvenes asocian el uso de casco con una identidad insegura, vulnerable o temerosa se resisten a usarlo para evitar la crítica de sus amigos ${ }^{(19),(20)}$.

En cuanto a los factores contextuales o ambientales, el uso de casco se ha asociado con diversas variables, como las zonas de circulación, los momentos del día y de la semana, las estaciones del año y las condiciones climáticas ${ }^{(6),(20),(21),(22)}$. En estudios previos realizados en Argentina, se ha encontrado un mayor uso de casco durante los días de semana, en condiciones climáticas adversas, durante el día, y en zonas céntricas ${ }^{(13),(14),(21)}$. No obstante, estos factores parecerían estar moderados por los contextos geográficos o culturales, pues los hallazgos de la literatura previa no son necesariamente consistentes. Por ejemplo, un estudio realizado en China no reflejó diferencias significativas de acuerdo al día de la semana, el momento del día o las condiciones climáticas ${ }^{(9)}$. En ese trabajo, el único factor contextual asociado al uso fue el tipo de camino, observándose un incremento del uso cuando se transitaba por calles de la ciudad, en comparación con caminos interurbanos o rurales. Los resultados sobre el uso de casco en distintas estaciones del año también son contradictorios. Por ejemplo, mientras algunos trabajos informaron una disminución durante el verano ${ }^{(20)}$ otros informan un patrón contrario ${ }^{(6)}$.

Entre los factores que se asocian al uso de casco, también merece una mención especial la presencia del Estado mediante acciones de control en las calles. La presencia o ausencia de control policial permitiría entender las variaciones en el uso de casco entre sectores periféricos y céntricos de la ciudad, así como también entre zonas rurales y urbanas ${ }^{(9),(13)}$. Por ejemplo, en un estudio reciente se observó que en zonas internas de la ciudad, donde existe control, la prevalencia de uso de casco es mucho mayor que en zonas externas sin vigilancia ${ }^{(23)}$. De modo similar, en México se compararon las tasas de uso de casco en cuatro zonas metropolitanas y los resultados indicaron que el lugar de mayor uso correspondía con la aplicación de acciones de control y sanción ${ }^{(11)}$. En otro estudio también se encontró que el incremento de los agentes de tránsito en la ciudad disminuyó las tasas de mortalidad ${ }^{(24)}$. En síntesis, las acciones de control y sanción parecen tener una función importante para entender los cambios en las tasas de uso de casco.

Dada la relevancia de esta medida de seguridad, Argentina ha incrementado los controles, las campañas de concientización y las acciones educativas en diferentes regiones del país ${ }^{(2)}$. Algunos estudios observacionales muestran el resultado de estos esfuerzos al destacar un incremento progresivo en la tasa de uso de $\operatorname{casco}^{(2),(21)}$. No obstante, estos trabajos no analizan sistemáticamente los cambios a través del tiempo ni permiten comparar resultados debido a diferencias metodológicas en la forma de relevar los datos. El valor de un estudio que muestre la evolución del uso de casco radica en la posibilidad de conocer concretamente cómo varían las tasas de uso a lo largo de los años y también determinar cambios en los factores asociados a esta conducta. Permitiría, a su vez, inferir los efectos que han tenido las intervenciones realizadas en términos de seguridad vial, y establecer si es necesario intensificar, modificar o planificar nuevas estrategias.

El objetivo de este trabajo es mostrar la evolución en el porcentaje de uso de casco en motociclistas en el período 2006-2014 en una ciudad argentina (Mar del Plata). Al mismo tiempo, se busca conocer los posibles factores asociados al uso. Este trabajo tiene la ventaja de que los datos han sido obtenidos siguiendo la misma metodología a través del tiempo. Los datos del año 2014 son inéditos y se informan aquí por primera vez. Los datos de años anteriores han dado lugar a publicaciones parciales ${ }^{(13),(14),(21)}$ y se utilizan en este caso con fines comparativos.

\section{MÉTODO}

\section{Muestra}

La población bajo estudio corresponde a conductores y pasajeros de moto de la ciudad 
de Mar del Plata (Argentina). La muestra actual (año 2014) está constituida por 2.466 conductores y 501 pasajeros, observados en 17 puntos de la ciudad (ver procedimiento para más detalle). Con fines comparativos, se utilizaron datos de muestras anteriores: 2006 $(n=962), 2008(n=977)$, y $2012(n=2.542)$. Cabe mencionar que en el año 2010 no se realizó el estudio, por este motivo no se cuenta con datos para ese año.

\section{Instrumento}

La metodología a través de los años no ha variado sustancialmente. Las observaciones se registraron siguiendo una planilla de codificación estandarizada, que contiene información sobre los momentos (fecha, hora, etc.) y lugares de observación; las condiciones ambientales y climáticas (hora, luz, temperatura, humedad, visibilidad, viento, estado del tiempo); las características de los vehículos (tipo de moto y presencia de patente); y de los usuarios (género y función). Para cada motociclista y acompañante se registró el uso o no uso de casco.

\section{Procedimiento}

En el año 2014 se siguió el mismo procedimiento que en las investigaciones anteriores. Las observaciones se realizaron en 17 lugares de la ciudad, similares a aquellos utilizados en estudios previos, sobre todo, en las observaciones del año 2012. Los sitios fueron principalmente urbanos, correspondientes al centro y macrocentro de la ciudad $(n=12)$, aunque se han considerado algunos puntos periféricos $(n=5)$. En cada sitio se realizaron observaciones en cuatro condiciones: de día y de noche (con luz artificial), y un día de semana y de fin de semana (sábado o domingo). En cada condición se completó una cuota mínima de observaciones $(n=35)$ que llevaron un tiempo aproximado de una hora cada una. En todos los casos se trató de intersecciones de vías. Las observaciones fueron realizadas entre los meses de julio y noviembre, en dos estaciones del año: invierno $(56 \%)$ y primavera $(44 \%)$.

Las observaciones fueron codificadas de acuerdo con la planilla estandarizada mencionada más arriba y fueron analizadas con el programa SPSS. Para el presente estudio se analizaron las tasas de uso de casco para el año 2014. Para estimar los factores asociados al uso de casco en conductores y pasajeros durante ese año se realizaron dos análisis de regresión logística con la variable uso/no uso como dependiente. Se utilizaron como factores predictores las siguientes variables: género, uso de casco en pasajeros o en conductores (dependiendo de la muestra objeto de análisis), condiciones climáticas, tipo de moto, momento del día, parte de la semana, presencia de patente, estación del año, e interacción entre momento del día y parte de la semana. Posteriormente, se estudió el porcentaje de uso de casco a lo largo de los años, desagregando la información por género y función (conductor o pasajero de la moto). Como para el período 2010 no se realizaron observaciones, se estimó el porcentaje promediando los períodos anterior y posterior.

\section{RESULTADOS}

\section{Prevalencia del uso de casco y factores asociados en el año 2014}

Los resultados muestran que el $81,3 \%$ de los conductores usan casco, mientras que en los pasajeros el porcentaje de uso disminuye al $57,7 \%$. En el Cuadro 1 se puede observar la prevalencia de uso de casco en diferentes condiciones.

Tal como se observa en el Cuadro 2 y el Cuadro 3, el análisis de regresión logística indica que el sexo se asocia significativamente al uso de casco en ambas muestras, conductores y pasajeros: su uso se reduce cuando el ocupante es varón. El uso de casco por parte de uno de los ocupantes también se asoció al uso en el otro ocupante, dicho de otro modo, cuando uno de ellos no usa casco se tiende a observar que el otro tampoco lo hace. 
Cuadro 1. Porcentaje de uso de uso de casco en conductores y pasajeros de motocicletas, según variables seleccionadas. Ciudad de Mar del Plata, Argentina, 2014.

\begin{tabular}{|c|c|c|c|c|}
\hline \multirow[t]{2}{*}{ Variables } & \multicolumn{2}{|c|}{ Conductores } & \multicolumn{2}{|c|}{ Pasajeros } \\
\hline & $\begin{array}{l}\text { Frecuencia } \\
(n=2466)\end{array}$ & $\begin{array}{c}\text { Uso de casco } \\
(\%)\end{array}$ & $\begin{array}{l}\text { Frecuencia } \\
\quad(n=501)\end{array}$ & $\begin{array}{c}\text { Uso de casco } \\
(\%)\end{array}$ \\
\hline \multicolumn{5}{|l|}{ Sexo } \\
\hline Varones & 2.235 & 78,7 & 180 & 42,8 \\
\hline Mujeres & 224 & 92,9 & 318 & 66,4 \\
\hline No identificado & 7 & 100,0 & 3 & 33,3 \\
\hline \multicolumn{5}{|l|}{ Clima } \\
\hline Despejado & 1.129 & 80,2 & 239 & 64,0 \\
\hline Nublado & 984 & 80,1 & 193 & 55,4 \\
\hline Lluvioso & 353 & 79,6 & 69 & 42,0 \\
\hline \multicolumn{5}{|l|}{ Tipo de Vehículo } \\
\hline Tipo “Biz” & 1.057 & 81,5 & 202 & 55,4 \\
\hline Ciclomotor/Scooter & 132 & 89,4 & 22 & 63,6 \\
\hline Calle & 668 & 79,2 & 155 & 60,6 \\
\hline Custom/Touring/Sport & 175 & 85,1 & 33 & 72,7 \\
\hline Off-road (Cross, Enduro, Trail, etc.) & 397 & 72,0 & 84 & 48,8 \\
\hline Otras & 22 & 81,8 & 2 & 50,0 \\
\hline No identificado & 15 & 93,3 & 3 & 100,0 \\
\hline \multicolumn{5}{|l|}{ Momento del día } \\
\hline Día & 1.259 & 81,7 & 237 & 56,1 \\
\hline Noche & 1.207 & 78,4 & 264 & 59,1 \\
\hline \multicolumn{5}{|l|}{ Momento de la Semana } \\
\hline Semana & 1.254 & 79,7 & 246 & 53,7 \\
\hline Fin de semana & 1.212 & 80,5 & 255 & 61,6 \\
\hline \multicolumn{5}{|l|}{ Presencia de patente } \\
\hline Sí & 2.234 & 81,5 & 437 & 59,0 \\
\hline No & 172 & 64,0 & 43 & 37,2 \\
\hline No identificado & 60 & 75,0 & 21 & 71,4 \\
\hline \multicolumn{5}{|l|}{ Estación del año } \\
\hline Invierno & 1.380 & 81,3 & 251 & 51,0 \\
\hline Primavera & 1.086 & 78,5 & 250 & 64,4 \\
\hline
\end{tabular}

Fuente: Elaboración propia.

Con relación a las variables del vehículo, tanto el tipo de moto como la presencia de patente se asociaron al uso del casco, aunque solo en la muestra de conductores. El uso de casco disminuye cuando se trata de modelos tipo Cross, Enduro o Trail, en comparación con la típica moto urbana (modelo "Biz"). Por su parte, los conductores que transitan en motos sin patente tienden a usar menos el casco, en comparación con los que conducen motos con patente.
Finalmente, entre las variables contextuales, la estación del año se asoció con diferencias en el uso de casco (solo en la muestra de conductores), con un aumento durante el invierno. Tanto en conductores como en pasajeros no se encontraron asociaciones entre el uso de casco y las condiciones climáticas, el momento del día, o la parte de la semana. Tampoco se observaron asociaciones entre el uso de casco y la interacción entre momento del día y parte de la semana. 
Cuadro 2. Factores asociados con el uso de casco en conductores de motocicleta. Análisis de regresión logística múltiple. Ciudad de Mar del Plata, Argentina, 2014.

\begin{tabular}{|c|c|c|}
\hline Variables & OR & IC95\% \\
\hline \multicolumn{3}{|l|}{ Sexo } \\
\hline Mujeres $^{1}$ & - & - \\
\hline Varones & 0,309 & 0,$172 ; 0,555$ \\
\hline \multicolumn{3}{|l|}{ Uso de casco en pasajeros } \\
\hline Sin pasajeros ${ }^{1}$ & - & - \\
\hline Pasajeros sin casco & 0,117 & 0,$075 ; 0,182$ \\
\hline Pasajeros con casco & 1,193 & 0,$836 ; 1,703$ \\
\hline \multicolumn{3}{|l|}{ Condiciones climáticas } \\
\hline Soleado $^{1}$ & - & - \\
\hline Nublado & 0,954 & 0,$680 ; 1,340$ \\
\hline Lluvioso & 0,815 & 0,$524 ; 1,268$ \\
\hline \multicolumn{3}{|l|}{ Tipo de vehículo } \\
\hline Tipo "Biz"1 & - & - \\
\hline Ciclomotor/Scooter & 1,914 & 0,$990 ; 3,701$ \\
\hline Calle & 0,923 & 0,$698 ; 1,220$ \\
\hline Custom/Touring/Sport & 1,538 & 0,$902 ; 2,622$ \\
\hline Off-road (Cross, Enduro, Trail, etc.) & 0,631 & 0,$465 ; 0,856$ \\
\hline \multicolumn{3}{|l|}{ Momento del día } \\
\hline Con luz ${ }^{1}$ & - & - \\
\hline Sin luz & 0,779 & 0,$561 ; 1,081$ \\
\hline \multicolumn{3}{|l|}{ Momento de la semana } \\
\hline Fin de semana $^{1}$ & - & - \\
\hline Semana & 1,337 & 0,$884 ; 2,021$ \\
\hline \multicolumn{3}{|l|}{ Presencia de patente } \\
\hline $\mathrm{S}^{\prime}{ }^{1}$ & - & - \\
\hline No & 0,404 & 0,$278 ; 0,587$ \\
\hline \multicolumn{3}{|l|}{ Estación del año } \\
\hline Primavera $^{1}$ & - & - \\
\hline Invierno & 1,794 & 1,$243 ; 2,589$ \\
\hline
\end{tabular}

\section{Evolución del uso de casco}

Los resultados indican un crecimiento progresivo en los porcentajes observados de uso de casco. La Figura 1 muestra los resultados desagregados por género y función (conductores y pasajeros). Puesto que el periodo 2008-2012 es más amplio que el resto, se incluyen estimaciones para el año 2010 a los efectos de facilitar la comparación bianual. Estas estimaciones se realizaron promediando los porcentajes del periodo anterior y posterior. Globalmente, se aprecia un aumento del uso de casco en todos los subgrupos, aunque el uso es siempre menor entre los pasajeros, en comparación con los conductores, y en los varones en comparación con las mujeres. El grupo más resistente al uso de casco es el de los varones que circulan como pasajeros. 
Cuadro 3. Factores asociados con el uso de casco en pasajeros de motocicleta. Análisis de regresión logística múltiple. Ciudad de Mar del plata, Argentina, 2014.

\begin{tabular}{|c|c|c|}
\hline Variables & OR & IC95\% \\
\hline \multicolumn{3}{|l|}{ Sexo } \\
\hline Mujeres $^{1}$ & - & - \\
\hline Varones & 0,391 & 0,$249 ; 0,614$ \\
\hline \multicolumn{3}{|l|}{ Uso de casco en conductores } \\
\hline Con casco ${ }^{1}$ & - & - \\
\hline Sin casco & 0,123 & 0,$077 ; 0,195$ \\
\hline \multicolumn{3}{|l|}{ Condiciones climáticas } \\
\hline Soleado $^{1}$ & - & - \\
\hline Nublado & 0,864 & 0,$442 ; 1,693$ \\
\hline Lluvioso & 0,392 & 0,$164 ; 0,939$ \\
\hline \multicolumn{3}{|l|}{ Tipo de vehículo } \\
\hline Tipo "Biz"1 & - & - \\
\hline Ciclomotor/Scooter & 1,193 & 0,$414 ; 3,436$ \\
\hline Calle & 1,163 & 0,$691 ; 1,957$ \\
\hline Custom/Touring/Sport & 1,930 & 0,$719 ; 5,181$ \\
\hline Off-road (Cross, Enduro, Trail, etc.) & 0,841 & 0,$449 ; 1,574$ \\
\hline \multicolumn{3}{|l|}{ Momento del día } \\
\hline Con luz ${ }^{1}$ & - & - \\
\hline Sin luz & 1,216 & 0,$650 ; 2,273$ \\
\hline \multicolumn{3}{|l|}{ Momento de la semana } \\
\hline Fin de semana ${ }^{1}$ & - & - \\
\hline Semana & 0,941 & 0,$437 ; 2,029$ \\
\hline \multicolumn{3}{|l|}{ Presencia de patente } \\
\hline $\mathrm{S}^{\prime}{ }^{1}$ & - & - \\
\hline No & 0,913 & 0,$412 ; 2,021$ \\
\hline \multicolumn{3}{|l|}{ Estación del año } \\
\hline Primavera $^{1}$ & - & - \\
\hline Invierno & 0,832 & 0,$407 ; 1,699$ \\
\hline
\end{tabular}

Fuente: Elaboración propia.

${ }^{1}$ Valor de referencia.

OR=odds ratio; IC95\%=intervalo de confianza del 95\%.

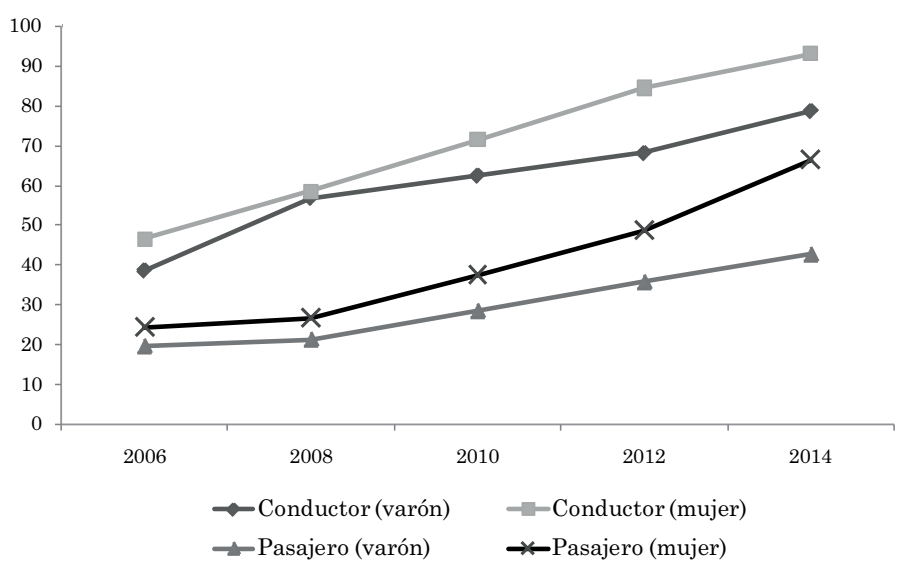

Figura 1. Evolución del porcentaje de uso de casco en conductores y pasajeros de acuerdo al sexo. Ciudad de Mar del Plata, Argentina, 2014.

Fuente: Elaboración propia. 


\section{DISCUSIÓN}

La información sobre comportamientos de riesgo en usuarios viales resulta clave para evaluar políticas de seguridad vial. Si, además, los datos permiten describir patrones de conducta a lo largo del tiempo, el valor en términos preventivos y de monitoreo de acciones es mayor. Una de las fortalezas del presente estudio es que ha permitido describir la evolución de un comportamiento preventivo (uso de casco en motociclistas) durante un período prolongado de tiempo (ocho años).

\section{Factores asociados al uso de casco}

Los resultados mostraron que las mujeres utilizan el casco en mayor medida que los varones, ya sea como conductoras o pasajeras, en coincidencia con lo observado en estudios previos realizados en nuestra ciudad ${ }^{(13),(14)}$ y en otros lugares ${ }^{(6),(9),(22)}$, y en línea con la idea general de que los varones tienden a mostrar mayores indicadores de riesgo en el tránsito ${ }^{(25)}$. Por otro lado, la función del ocupante de la moto también se relaciona con el uso de casco: los pasajeros presentan una tasa mucho menor de utilización de casco en comparación con los conductores. Este resultado es similar a otros estudios ${ }^{(11),(21)}$, y se relaciona principalmente con la falta de disponibilidad de un segundo casco en viajes con pasajeros que, en muchos casos, son ocasionales.

En cuanto a las variables del vehículo, se siguen observando diferencias en función del tipo de moto utilizada, lo que probablemente se asocia a perfiles diferentes de usuarios de moto $^{(13),(14),(21)}$. Asimismo, persiste la asociación entre uso de casco y presencia de patente en la moto ${ }^{(13),(14),(21)}$. Este resultado también es coherente con estudios de otros países $^{(9)}$. Creemos que esta relación se podría explicar en parte por un factor común: la predisposición de los conductores a cumplir con las normas de tránsito ${ }^{(13),(14)}$.

En cuanto a las variables contextuales, se observa que ahora tienen un peso menor que en estudios previos ${ }^{(13),(14),(21)}$. Solo se encontraron diferencias para el momento del año (mayor uso en invierno), en concordancia con estudios de otros lugares ${ }^{(6)}$. Globalmente, estos resultados sugieren que el uso en nuestra ciudad ha pasado a ser más consistente o menos dependiente de factores tales como el momento del día o la semana, o la situación climática.

\section{Evolución del uso de casco}

El casco es un elemento básico para la protección de los motociclistas, por ello toda estrategia de prevención y promoción de la seguridad en este grupo contempla iniciativas tendientes a controlar y promover su uso. En Argentina, estas iniciativas se han extendido e intensificado en los últimos tiempos, tanto a nivel nacional como a nivel de las localidades. En el marco del Plan Estratégico de Seguridad Vial impulsado por la Agencia Nacional de Seguridad Vial ${ }^{(2)}$ se han propuesto actividades diversas que apuntan a remarcar el valor del casco como elemento central en el resguardo de la vida del motociclista. Se destaca el desarrollo de un aula virtual que ofrece un curso gratuito con información relevante para motociclistas. Simultáneamente, en la ciudad de Mar del Plata se han profundizado los controles policiales y la aplicación de sanciones ante la falta de uso ${ }^{(26)}$. A las acciones de control se sumaron campañas que buscan concientizar a los motociclistas sobre comportamientos de autoprotección. Una acción importante asociada a los controles ha sido la entrega gratuita de cascos para quienes circulan sin usarlo y no poseen uno ${ }^{(27)}$. Otra iniciativa en la misma dirección ha sido establecer la obligatoriedad de entregar un casco homologado al vender una moto, y corroborar que el comprador posea licencia de conducir y seguro $^{(28)}$.

Los resultados del presente estudio muestran que, cuando las políticas de control y educación se sostienen en el tiempo, se puede mejorar progresivamente la tasa de uso de casco en una comunidad. Aún así, 
persiste el desafío de desarrollar estrategias de comunicación, concientización y educación que lleguen a los segmentos de usuarios más renuentes a utilizar el casco, por ejemplo, los pasajeros de moto. Por otro lado, si bien los datos a través del tiempo muestran una evolución favorable, es necesario contextualizar los resultados y considerar un indicador menos alentador como es el aumento del parque de motos. Como lo indican los datos de la Agencia Nacional de Seguridad Vial ${ }^{(2)}$, mientras en el año 2008 el tamaño del parque era de 2.500.000 unidades, en el año 2011 se registraron aproximadamente 4.200.000 motos. Curiosamente, en el período 2008-2011 el incremento del parque de motos fue cinco veces superior en comparación con el parque automotor.

En este contexto, parece imprescindible comenzar a pensar estrategias de acción que contemplen cambios más estructurales en los modos y patrones de movilidad, y que permitan desincentivar la compra y uso de motos. Entendemos que se requieren más esfuerzos tendientes a la promoción y el mejoramiento del transporte público, para que constituya una verdadera opción frente a las formas individuales y motorizadas de movilidad. Asimismo, también sería importante incentivar las formas activas de transporte (por ejemplo, el uso de bicicleta), como alternativas más saludables, económicas y ambientalmente sustentables. A nuestro juicio, el desafío a futuro será integrar las políticas de seguridad vial a planes más ambiciosos de planificación en materia de movilidad urbana.

\section{Limitaciones del estudio}

Por último, es importante señalar algunas limitaciones del presente estudio. En primer lugar, cabe mencionar que no se realizaron observaciones durante el verano, lo que podría afectar la estimación de la prevalencia de uso de casco. Estudios previos señalan una disminución del uso en esta época del año ${ }^{(20)}$, aunque la evidencia al respecto no es concluyente $^{(6)}$. En particular, la ciudad de Mar del Plata constituye una zona turística que en verano recibe una proporción considerable de visitantes. Esto provoca un cambio determinante en el contexto vial que se refleja en el aumento del parque de motos y el incremento de incidentes en motociclistas ${ }^{(29)}$. En este sentido, debido a la idiosincrasia de esta ciudad se podría suponer que la realización de observaciones durante el verano permitiría encontrar mayores diferencias entre las estaciones del año.

En segundo lugar, pueden existir algunos problemas de representatividad en las muestras trabajadas. Como ya se ha mencionado, los sitios de observación cubrieron principalmente el centro y macrocentro de la ciudad, siendo mucho menor la cantidad de puntos de observación en zonas periféricas. Tampoco se incluyeron puntos suburbanos o rurales. Esto podría generar una sobreestimación en la prevalencia de uso de casco, si se tiene en cuenta que algunos estudios previos muestran una reducción del uso tanto en zonas periféricas como rurales ${ }^{(9),(13)}$. Por último, otro sesgo podría relacionarse con los momentos del día en que se hicieron las observaciones. El hecho de no encontrar diferencias entre el día y la noche, como en otros estudios ${ }^{(22)}$, se podría deber a que nuestras observaciones no cubrieron toda la noche sino que fueron realizadas entre las 20 y las 22 hs., aproximadamente. Es posible que, en horas de la madrugada, se produzca una reducción en el uso del casco, que no haya sido detectada.

Finalmente, es necesario señalar que, si bien los resultados de este estudio son útiles para conocer factores asociados al uso de casco, la progresión de uso en un período prolongado de tiempo y la identificación de los grupos que requieren más atención no brindan conocimientos sobre las motivaciones de los individuos para utilizar esta medida de autoprotección. Si bien existen estudios previos que destacan el papel de las actitudes, sobre todo del componente emocional y de las evaluaciones implícitas ${ }^{(16),(17)}$, sigue siendo necesario estudiar los aspectos psicológicos y comportamentales que influyen en el uso del casco. 


\section{REFERENCIAS BIBLIOGRÁFICAS}

1. Organización Mundial de la Salud. Informe sobre la situación mundial de la seguridad vial [Internet]. 2013 [citado 10 mar 2015]. Disponible en: http://goo.gl/YOVV3P.

2. Agencia Nacional de Seguridad Vial. Plan estratégico de seguridad vial para motovehículos [Internet]. 2012 [citado 10 mar 2015]. Disponible en: http://goo.gl/HjSfRn.

3. Yusuf AS, Odebode TO, Adeniran JO, Salaudeen AG, Adeleke NA, Alimi MF. Pattern and outcome of motorcyclists head injury in Ilorin, Nigeria. Nigerian Journal Basic Clinical Sciences. 2014;11(2):80-84.

4. Yu WY, Chen CY, Chiu WT, \& Lin MR. Effectiveness of different types of motorcycle helmets and effects of their improper use on head injuries. International Journal of Epidemiology. 2011; doi: 10.1093/ije/dyr040.

5. Fernandes FAO, Alves de Sousa RA. Motorcycle helmets: A state of the art review. Accident, Analysis and Prevention. 2013;56:1-21.

6. Papadakaki M, Tzamalouka G, Orsi C, Kritikos A, Morandi A, Gnardellis C, Chliaoutakis J. Barriers and facilitators of helmet use in a Greek sample of motorcycle riders: Which evidence? Transportation Research Part F: Traffic Psychology and Behavior. 2013;18:189-198.

7. Ramli R, Oxley J, Hillard P, Sadullah AFM, \& McClure $R$. The effect of motorcycle helmet type, components and fixation status on facial injury in Klang Valley, Malaysia: a case control study. BMC Emergency Medicine. 2014; doi: 10.1186/1471-227X-14-17.

8. Crompton JG, Oyetunji TA, Pollack KM, Stevens $\mathrm{K}$, Cornwell EE, Efron DT, Haider AH. Association between helmets and facial injury after a motorcycle collision: an analysis of more than 40000 patients from the National Trauma Data Bank. Archives of Surgery. 2012;147(7):674-676

9. Xuequn $Y$, Ke L, Ivers R, Du W, Senserrick T. Prevalence rates of helmet use among motorcycle riders in a developed region in China. Accident, analysis and prevention. 2011;43(1):214-219.

10. Brown CV, Hejl K, Bui E, Tips G, Coopwood B. Risk factors for riding and crashing a motorcycle unhelmeted. Journal of Emergency Medicine. 2011;41(4):441-446.

11. Trejo AC, Leenen I. Uso del casco por parte de los motociclistas de cuatro zonas metropolitanas de la República Mexicana. Gaceta Médica de México. 2014;150(Supl 1):11-20.

12. Bachani AM, Tran NT, Sann S, Ballesteros MF, Gnim C, Ou A, Hyder AA. Helmet use among motorcyclists in Cambodia: A survey of use, knowledge, attitudes, and practices. Traffic Injury Prevention. 2012;13(1):31-36.

13. Ledesma RD, Peltzer RI. Helmet use among motorcyclists: Observational study in the city of Mar del Plata, Argentina. Revista de Saúde de Pública. 2008;42(1):143-145.
14. Ledesma RD, Peltzer RI, Poó FM, Cremonte M. Motorcycle helmet use in Mar del Plata, Argentina. In: Argosyan VE, (ed). Protective devices: types, uses and safety. New York: Nova Science Publisher; 2010.

15. Brijs K, Brijs T, Sann S, Trinh TA, Wets G, Ruiter RA. Psychological determinants of motorcycle helmet use among young adults in Cambodia. Transportation Research Part F: Traffic Psychology and Behavior. 2014;26:273-290.

16. Orsi C, Stendardo A, Marinoni A, Gilchrist MD, Otte D, Chliaoutakis J, Morandi A. Motorcycle riders' perception of helmet use: Complaints and dissatisfaction. Accident, Analysis and Prevention. 2012;44(1):111-117.

17. Ledesma RD, Tosi JD, Poó FM, Montes SA, López SS. Implicit attitudes and road safety behaviors: The helmet-use case. Accident, Analysis and Prevention. 2015;79:190-197.

18. Ranney ML, Mello MJ, Baird JB, Chai PR, Clark MA. Correlates of motorcycle helmet use among recent graduates of a motorcycle training course. Accident, Analysis and Prevention. 2010;42(6):2057-2062.

19. Fuentes C, Gras ME, Font-Mayolas S, Bertran C, SuIIman MJ, Ballester D. Expectations of efficacy, social influence and age as predictors of helmet-use in a sample of Spanish adolescents. Transportation Research Part F: Traffic Psychology and Behavior. 2010;13(5):289-296.

20. Zamani-Alavijeh F, Bazargan M, Shafiei A, Bazargan-Hejazi $S$. The frequency and predictors of helmet use among Iranian motorcyclists: A quantitative and qualitative study. Accident, Analysis and Prevention. 2011;43(4):1562-1569.

21. Ledesma RD, López SS, Tosi JD, Poó FM. Motorcycle Helmet use in Mar del Plata, Argentina: prevalence and associated factors. International Journal of Injury Control and Safety Promotion. 2014;22(2):172-176.

22. Akaateba MA, Amoh-Gyimah R, Yakubu I. A crosssectional observational study of helmet use among motorcyclists in Wa, Ghana. Accident, Analysis and Prevention. 2014;64:18-22.

23. Karuppanagounder K, Vijayan AV. Motorcycle helmet use in Calicut, India: users behaviour, attitude and perceptions. Traffic Injury Prevention. 2015; doi: 10.1080/15389588.2015.1055736.

24. Espitia-Hardeman V, Vélez L, Muñoz E, GutiérrezMartínez MI, Espinosa-Vallín R, Concha-Eastman A. Efectos de las intervenciones diseñadas para prevenir las muertes de motociclistas en Cali, Colombia (1993-2001). Salud Pública de México. 2008;50:69-77.

25. Rhodes N, Pivik K. Age and gender differences in risky driving: the roles of positive affect and risk perception. Accident, Analysis and Prevention. 2011;43:923-931.

26. Municipalidad de General Pueyrredón. El Municipio secuestró 72 motos por falta de casco en los últimos días [Internet]. 3 nov 2014 [citado 7 ago 2015]. Disponible en: http://goo.gl/DqTwHU. 
27. Municipalidad de General Pueyrredón. Nuevo operativo de control de motos y entrega de cascos [Internet]. 26 feb 2014 [citado 7 ago 2015]. Disponible en: http://goo.gl/EQPXXE

28. Municipalidad de General Pueyrredón. El Municipio comenzó con la campaña de difusión de las nuevas medidas de seguridad provinciales [Internet]. 15 abr 2014 [citado 7 ago 2015]. Disponible en: http://goo.gl/3GaUjc.
29. Municipalidad de General Pueyrredón. Balance de Movilidad Urbana Municipal de los controles preventivos de tránsito durante la temporada [Internet]. 20 feb 2014 [citado 7 ago 2015]. Disponible en: http://goo.gl/7TYHtz.

FORMA DE CITAR

Tosi JD, Ledesma RD, Poó FM, Montes SA, López SS. Prevalencia y evolución del uso de casco en ocupantes de motocicletas en una ciudad argentina (Mar del Plata, 2006-2014). Salud Colectiva. 2016;12(1):85-95.

Recibido: 22 de mayo de 2015 | Versión final: 25 de agosto de 2015 | Aprobado: 16 de octubre de 2015

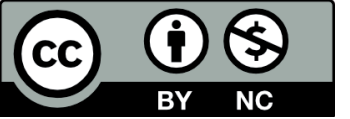

Este obra está bajo una licencia de Creative Commons Reconocimiento-NoComercial 4.0 Internacional. Reconocimiento - Permite copiar, distribuir y comunicar públicamente la obra. A cambio, se debe reconocer y citar al autor original. No Comercial - Esta obra no puede ser utilizada con finalidades comerciales, a menos que se obtenga el permiso.

http://dx.doi.org/10.18294/sc.2016.679 